\title{
Twist-related protein 1 enhances oral tongue squamous cell carcinoma cell invasion through $\boldsymbol{\beta}$-catenin signaling
}

\author{
LIAN ZHENG, NING LI, FENG GUO, XIN-CHUN JIAN, CAN-HUA JIANG, \\ PING YIN, AN-JIE MIN and LONG HUANG \\ Department of Oral and Maxillofacial Surgery, Xiangya Hospital, \\ Central South University, Changsha, Hunan 410008, P.R. China
}

Received January 20, 2014; Accepted August 7, 2014

DOI: $10.3892 / \mathrm{mmr} .2014 .2904$

\begin{abstract}
Accumulating evidence suggests that $\beta$-catenin signaling may be involved in oral tongue squamous cell carcinoma (OTSCC) cell invasion. Abnormal activation of twist-related protein 1 (TWIST1 or TWIST) has been identified in several types of human cancer. A recent study showed that overexpression of TWIST is associated with a poor prognosis in patients with OTSCC and may enhance OTSCC cell invasion. This study investigated the effect of TWIST on $\beta$-catenin signaling in OTSCC cells and its impact on OSTCC cell invasion. Stable overexpression of TWIST, with or without knockdown of $\beta$-catenin, and stable knockdown of TWIST were performed in SCC-4 and TCA8113 human OTSCC cells. Overexpression of TWIST in SCC-4 and TCA8113 cells increased $\beta$-catenin signaling luciferase reporter activity, mRNA levels of the $\beta$-catenin signaling target genes, c-Myc and c-Jun levels, soluble $\beta$-catenin level, the phosphorylation status of glycogen synthase kinase-3 $\beta$ (GSK-3 $\beta$ ) at serine 9, matrix metalloproteinase-2 (MMP-2) expression and cell invasion. Knockdown of TWIST had the opposite effect. All of these changes, with the exception of phosphorylation of GSK-3 $\beta$, were eliminated by stable knockdown of $\beta$-catenin. In addition, the phosphatidylinositol 3-kinase (PI3K) inhibitor, LY294002 abrogated the enhancing effects of TWIST on mRNA levels of c-Myc and c-Jun, soluble $\beta$-catenin levels, MMP-2 expression, cell invasion and GSK-3 $\beta$ phosphorylation. In conclusion, the present study demonstrated that TWIST enhances cell invasion and MMP-2 expression in OTSCC cells through $\beta$-catenin signaling, probably via a PI3K-dependent mechanism. This study provides novel insights into the molecular mechanisms underlying OTSCC progression.
\end{abstract}

Correspondence to: $\mathrm{Dr} \mathrm{Ning} \mathrm{Li}$, Department of Oral and Maxillofacial Surgery, Xiangya Hospital, Central South University, 87 Xiangya Road, Changsha, Hunan 410008 P.R. China

E-mail: liningbeta@hotmail.com; lining463@yahoo.com

Key words: twist-related protein $1, \beta$-catenin, oral tongue squamous cell carcinoma, cell invasion, matrix metalloproteinase, phosphatidylinositol 3-kinase

\section{Introduction}

Oral squamous cell carcinoma is the most common form of head and neck cancer. It accounts for $>90 \%$ of all such cancers and has a poor prognosis that may be attributable to the high frequency of lymph node metastasis and local invasion (1). Tongue cancer is the most common form of intraoral cancer. Its incidence is rising in comparison with that of cancer in other head and neck sites (2). Metastatic tongue carcinoma is associated with poorer survival and a lower rate of local tumor control than other sites of head and neck cancer and has a five-year survival rate of just $50 \%$ (2). The development of oral tongue squamous cell carcinoma (OTSCC) metastasis currently poses significant clinical challenges due to the limited therapeutic options that are available (3).

Twist-related protein 1 (TWIST), also known as TWIST1, is a member of the basic helix-loop-helix transcription factor family. During embryonic development, TWIST is essential in the development of the mesoderm and differentiation of mesoderm-derived tissues (4). A high level of expression of TWIST has been detected in several forms of cancer and has been associated with the initial phase of metastatic progression (5). A recent study has shown that overexpression of TWIST is associated with a poor prognosis in patients with OTSCC and that knockdown of TWIST inhibits OTSCC cell invasion (6).

$\beta$-catenin, originally identified as an essential regulator of E-cadherin-mediated cell-cell interaction, is a key component of the Wnt signaling pathway (7). In the majority of cells, $\beta$-catenin is predominantly located at the plasma membrane in a complex with cadherins and $\alpha$-catenin. This forms the insoluble pool of $\beta$-catenin. Under normal conditions, a small quantity of soluble $\beta$-catenin, which is free from cadherin, is present in the cytoplasm (8). Wnt signals are transduced via specific cell surface receptors and activate a series of biochemical reactions, involving a large protein complex consisting of $\beta$-catenin and glycogen synthase kinase-3 $\beta$ (GSK-3 $\beta$ ). This results in stabilization of soluble $\beta$-catenin and therefore an increase in the soluble pool of this molecule (9). Soluble $\beta$-catenin interacts with $\mathrm{T}$ cell factor (Tcf) family transcription factors to activate a number of downstream target genes, such as c-Myc and c-Jun, which are important in the initiation and progression of carcinogenesis $(8,10,11)$. Recent studies have provided in vitro evidence 
that $\beta$-catenin signaling is pivotal in facilitating OTSCC cell invasion $(12,13)$.

A pilot study conducted by our group suggested that TWIST may regulate $\beta$-catenin signaling in OTSCC cells The current study investigated the effect of TWIST on $\beta$-catenin signaling in OTSCC cells and its impact on OSTCC cell invasion.

\section{Materials and methods}

Cells lines, plasmids and reagents. Human SCC-4 and TCA8113 OTSCC cell lines were obtained from the American Type Culture Collection (Manassas, VA, USA) and Wuhan Boster Bio-Engineering Inc. (Wuhan, China), respectively. Human Twist cDNA was subcloned into a pcDNA 3.1 expression vector (14). TOPflash and FOPflash plasmids were obtained from Millipore (Billerica, MA, USA). Twist (sc-38604-V) and $\beta$-catenin (sc-29209-V) short hairpin (sh) RNA lentiviral particles, control shRNA lentiviral particles-A (sc-108080), anti-TWIST (sc-81417) mouse monoclonal antibodies, anti- $\beta$-catenin (sc-7963) mouse monoclonal antibodies and anti-matrix metalloproteinase-2 (MMP-2, sc-53630) mouse monoclonal antibodies were obtained from Santa Cruz Biotechnology Inc. (Santa Cruz, CA, USA). Anti-GSK-3 $\beta$ and Anti-phospho-GSK-3 $\beta$ (serine 9) rabbit monoclonal antibodies were obtained from Cell Signaling Technology, Inc. (Beverly, MA, USA). Superfect ${ }^{\mathrm{TM}}$ transfection reagent was purchased from Qiagen (Valencia, CA, USA). A dual-luciferase reporter assay system was obtained from Promega Corporation (Madison, WI, USA). G418, puromycin, LY294002 and all chemicals of reagent grade were obtained from Sigma (St. Louis, MO, USA).

Transfection and lentiviral transduction. The TWIST expression construct was transfected into cells using Superfect ${ }^{\mathrm{TM}}$ transfection reagent (Qiagen) according to the manufacturer's instructions. Pools of stable transfectants were generated via selection with $\mathrm{G} 418(800 \mu \mathrm{g} / \mathrm{ml})$ according to the manufacturer's instructions. Lentiviral transduction was performed as previously described (15), and pools of stable transductants were generated via selection with puromycin $(5 \mu \mathrm{g} / \mathrm{ml})$.

Western blot analysis. Immunoblotting was performed with the appropriate antibodies. Soluble cell lysate fractions were prepared as previously described (15). Briefly, cells were lysed in $0.1 \%$ Nonidet P-40 lysis buffer $(0.1 \%$ Nonidet P-40; $10 \mathrm{mM}$ HEPES, pH 7.5; $142.5 \mathrm{mM} \mathrm{KCl;} 5 \mathrm{mM} \mathrm{MgCl}_{2}$; and $1 \mathrm{mM}$ ethylene glycol tetra acetic acid). The lysates were centrifuged at $14,000 \mathrm{x} \mathrm{g}$ for $10 \mathrm{~min}$ and the supernatants were saved as soluble cell lysate. To prepare the whole cell lysate, cells were dissolved in $250 \mu \mathrm{l}$ of $2 \mathrm{X}$ SDS loading buffer $(62.5 \mathrm{~mm}$ TrisHCl, pH 6.8; 2\% SDS; 25\% glycerol; $0.01 \%$ bromphenol blue; and $5 \%$ 2-mercaptoethanol), and incubated at $95^{\circ} \mathrm{C}$ for $10 \mathrm{~min}$. Equal quantities of proteins for each sample were separated by $10 \%$ SDS-polyacrylamide gel and blotted onto polyvinylidene difluoride microporous membranes (Millipore). Membranes were incubated for $1 \mathrm{~h}$ with a 1/1,000 dilution of primary antibody, and then washed and revealed using secondary antibodies conjugated to horseradish peroxidase $(1 / 5,000,1 \mathrm{~h})$.
Peroxidase was visualized with a GE Healthcare enhanced chemiluminescence kit (Beijing, China).

Reverse transcription-quantitative polymerase chain reaction $(R T-q P C R)$. RNA was prepared from cells using TRIzol reagent (Life Technologies, Carlsbad, CA, USA) followed by purification with a TURBO DNA-free system (Ambion, Austin, TX, USA). A total of $200 \mathrm{ng}$ cDNA was synthesized using SuperScript II reverse transcriptase (Invitrogen, Carlsbad, CA, USA). RT-qPCR was performed on the LightCycler thermal cycler system (Roche Diagnostics, Indianapolis, IN, USA) using SYBR Green I kit (Roche Diagnostics) according to the manufacturer's instructions. The PCR amplification conditions were as follows: $20 \mathrm{sec}$ at $95^{\circ} \mathrm{C}$ followed by 40 cycles of $3 \mathrm{sec}$ at $95^{\circ} \mathrm{C}$ and $30 \mathrm{sec}$ at $60^{\circ} \mathrm{C}$. The results were normalized against those of the reference gene, glyceraldehyde-3-phosphate dehydrogenase (GAPDH). The following primers were used: Forward: 5'-AGGGATTTTCTCAGTCCTTC-3' and reverse: 5'-CATGCCCTCATCTAATGTCT-3' for $\beta$-catenin; forward: 5'-GGACGACGAGACCTTCATCAA-3' and reverse: 5'-CCAGCTTCTCTGAGACGAGCTT-3' for human c-Myc; forward: 5'-CAAAGTTTGGATTGCATCAAGTG-3' and reverse: 5'-TAACATTATAAATGGTCACAGCACATG-3' for human c-Jun; and forward: 5'-GACTCATGACCA CAGTCCATGC-3' and reverse: 5'-AGAGGCAGGGATG ATGTTCTG-3' for human GAPDH. Each experiment was repeated twice in triplicate.

Luciferase assay. SCC-4 and TCA8113 cells were transfected with TOPflash or FOPflash plasmids using Superfect transfection reagent (Qiagen). PRL-CMV plasmid encoding Renilla reniformis luciferase (at a concentration of one fifth molar ratio relative to the test plasmids) was co-transfected as an internal control. The luciferase assays were performed following $24 \mathrm{~h}$ transfection with a dual-luciferase reporter assay system (Promega Corporation) according to the manufacturer's instructions. Each experiment was repeated three times in duplicate.

In vitro cell invasion assay. Transwell ${ }^{\circledR}$ cell-culture chambers with $8-\mu \mathrm{m}$ pores (BD Biosciences, Bedford, MA, USA) and 24 wells per plate were coated with $50 \mu \mathrm{l}$ Matrigel $(10 \mathrm{mg} / \mathrm{ml}$; BD Biosciences; diluted 1:3). SCC-4 and TCA8113 cells were seeded in the upper chamber at $5 \times 10^{5}$ cells per well, in Dulbecco's modified Eagle's medium and RPMI-1640 serum-free medium, respectively. Complete medium $(600 \mu \mathrm{l})$ with $10 \%$ fetal bovine serum was added to the lower chamber. After $24 \mathrm{~h}$ of incubation, cells were fixed and stained with crystal violet. Invading cells were counted in five random fields per chamber under an inverted microscope (IX83; Olympus, Bejing, China). Each experiment was repeated three times in duplicate.

Statistical analysis. Statistical analyses were performed using SPSS for Windows 10.0 (SPSS Inc., Chicago, IL, USA). Data are expressed as the mean \pm standard deviation. Comparisons of means between groups were performed with one-way analysis of variance followed by post hoc pairwise comparisons using Tukey's tests. $\mathrm{P}<0.05$ was considered to indicate a statistically significant difference. 
A
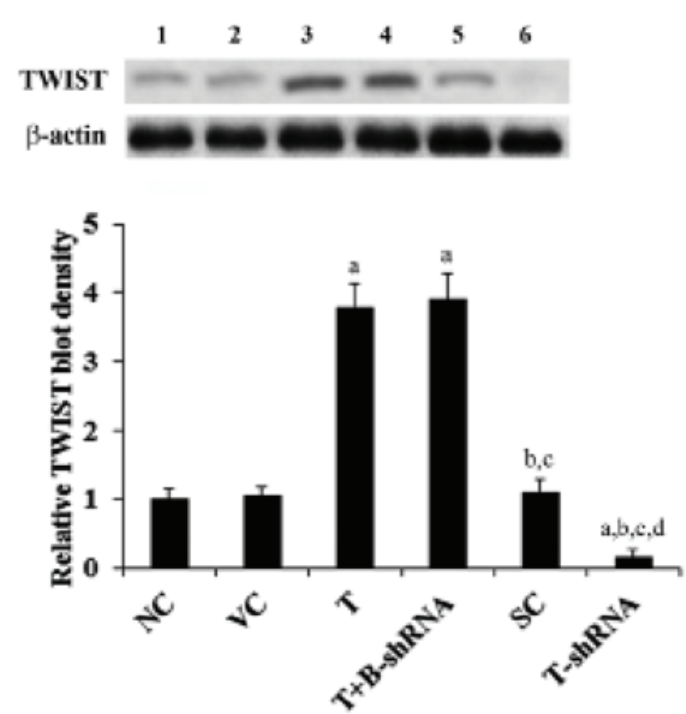
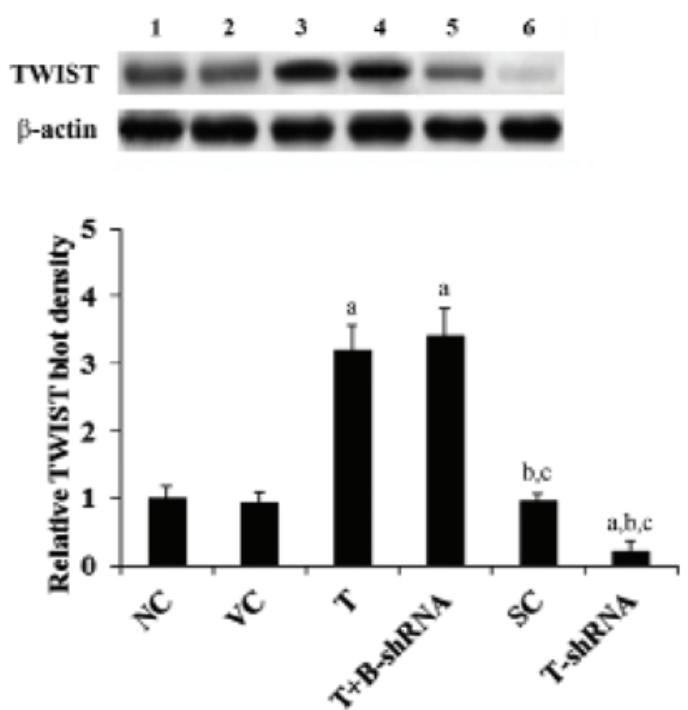

Figure 1. TWIST expression in OTSCC cells with overexpression and knockdown of TWIST. (A) SCC-4 and (B) TCA8113 human OTSCC cells. Lane 1, NC; lane 2, VC; lane 3, T; lane 4, T+B-shRNA; lane 5, SC; lane 6, T-shRNA. Expression was analyzed with western blot analysis. $\beta$-actin was used as a loading control. The density of the TWIST blots were normalized against that of $\beta$-actin blots to obtain a relative blot density. This was expressed as a fold change of the relative TWIST blot density compared with the $\mathrm{NC}$ group (designated as 1 ). ${ }^{\mathrm{a}} \mathrm{P}<0.05$, compared with $\mathrm{NC}$ or $\mathrm{VC},{ }^{\mathrm{b}} \mathrm{P}<0.05$, compared with $\mathrm{T}$, $\mathrm{P}<0.05$, compared with T+B-shRNA and ${ }^{\mathrm{d}} \mathrm{P}<0.05$, compared with SC. OTSCC, oral tongue squamous cell carcinoma; NC, normal control; VC, cells transfected with empty pcDNA3 vector; T, cells transfected with pcDNA3-TWIST expression vector; T+B-shRNA, cells transfected with pcDNA-TWIST expression vector and $\beta$-catenin short hairpin RNA; SC, cells transfected with scrambled control shRNA; T-shRNA, cells transfected with TWIST shRNA; TWIST, twist-related protein 1.

A

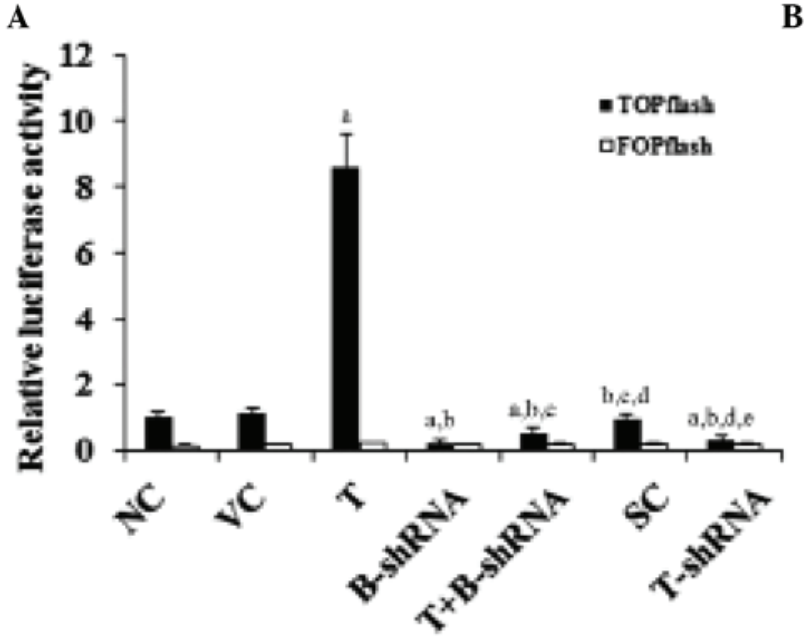

B

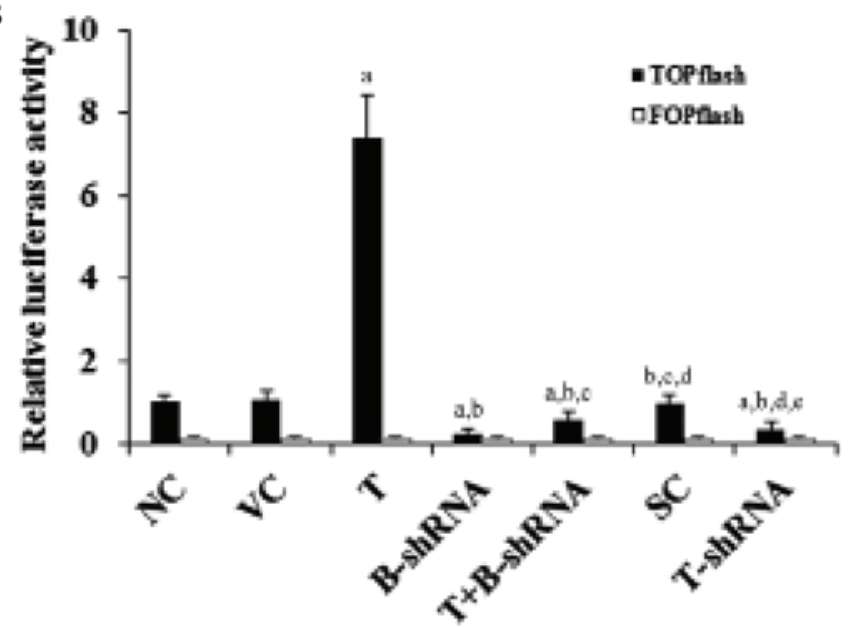

Figure 2. Effect of TWIST on $\beta$-catenin luciferase reporter activity in OTSCC cells. (A) SCC-4 and (B) TCA8113 human OTSCC cells were transfected with TOPflash, a synthetic $\beta$-catenin luciferase reporter, or FOPflash, a negative control reporter for TOPflash. After 24 h, the luciferase activity in each group was analyzed. The luciferase activity was expressed as a fold change relative to that of the NC group (designated as 1 ). ${ }^{a} \mathrm{P}<0.05$, compared with $\mathrm{NC}$ or $\mathrm{VC}$, ${ }^{\mathrm{b}} \mathrm{P}<0.05$, compared with $\mathrm{T},{ }^{\mathrm{C}} \mathrm{P}<0.05$, compared with $\mathrm{B}$-shRNA, ${ }^{\mathrm{d}} \mathrm{P}<0.05$, compared with $\mathrm{T}+\mathrm{B}$-shRNA and ${ }^{\mathrm{e}} \mathrm{P}<0.05$, compared with $\mathrm{SC}$. OTSCC, oral tongue squamous cell carcinoma; NC, normal control; VC, cells transfected with empty pcDNA3 vector; T, cells transfected with pcDNA3-TWIST expression vector; T+B-shRNA, cells transfected with pcDNAs-TWIST expression vector and $\beta$-catenin short hairpin RNA; SC, cells transfected with scrambled control shRNA; T-shRNA, cells transfected with TWIST shRNA; TWIST, twist-related protein 1.

\section{Results}

TWIST expression is increased by transfection with a TWIST expression vector and decreased by transduction of TWIST shRNA. To investigate the function of TWIST in OTSCC cells, SCC-4 and TCA8113 human OTSCC cells were stably transfected with a TWIST expression vector to induce overexpression of TWIST. In addition, a separate group of cells was stably transduced with TWIST-shRNA in order to knock down the expression of this gene. As shown in Fig. 1, TWIST is constitutively expressed in SCC- 4 and TCA8113 cells. Its expression was reduced by $\sim 80 \%$ by stable transduction of TWIST-shRNA. Compared with controls, TWIST expression was increased three-fold in SCC-4 and TCA8113 cells that had 
A

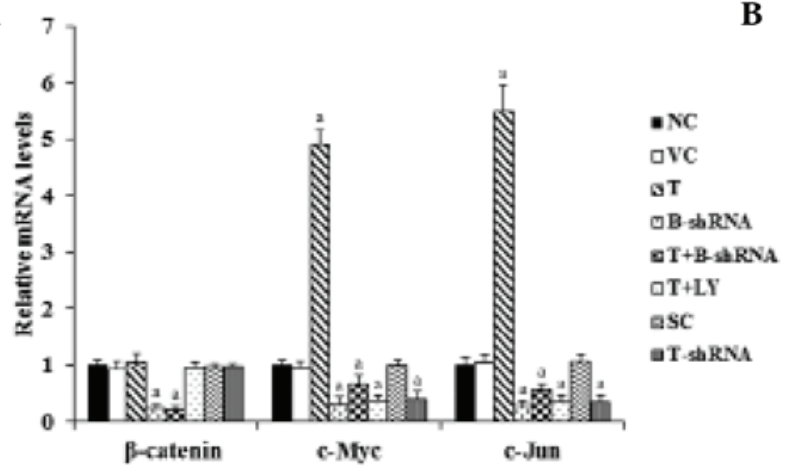

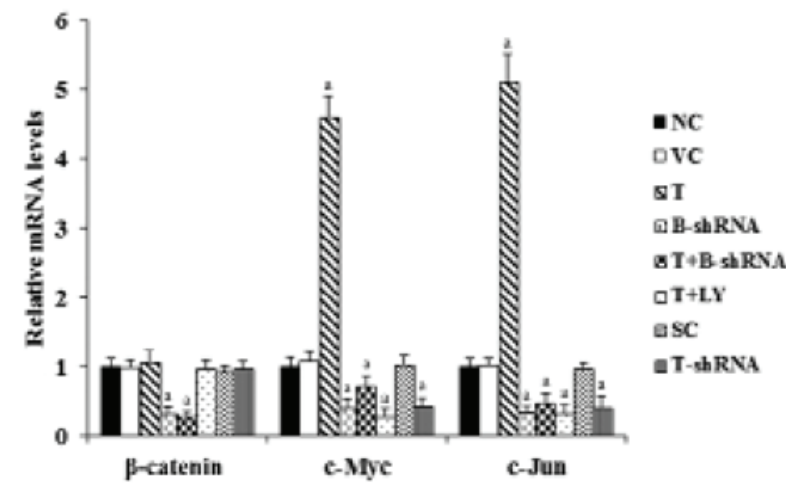

Figure 3. Effect of TWIST on mRNA levels of $\beta$-catenin, C-Myc and C-Jun in OTSCC cells. (A) SCC-4 and (B) TCA8113 human OTSCC. Reverse transcription-qunatitative polymerase chain reaction was performed in each group. The mRNA levels were expressed as a fold change relative to that of the NC group (designated as 1). ${ }^{a} \mathrm{P}<0.05$, compared with $\mathrm{NC}$ or VC. OTSCC, oral tongue squamous cell carcinoma; NC, normal control; VC, cells transfected with empty pcDNA3 vector; T, cells transfected with pcDNA3-TWIST expression vector; $\mathrm{T}+\mathrm{B}$-shRNA, cells transfected with pcDNAs-TWIST expression vector and $\beta$-catenin short hairpin RNA; T+Ly, cells transfected with pcDNAs-WIST expression vector and treated with $50 \mu \mathrm{m}$ LY294002; SC, cells transfected with scrambled control shRNA; T-shRNA, cells transfected with TWIST shRNA; TWIST, twist-related protein 1.

$\mathbf{A}$
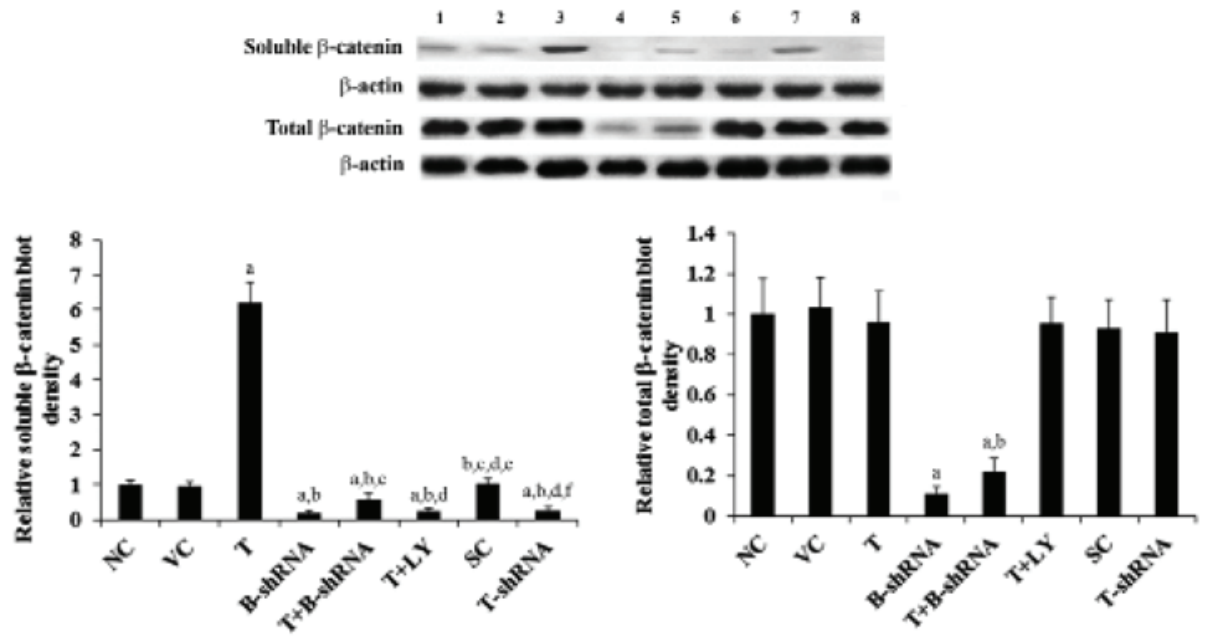

B
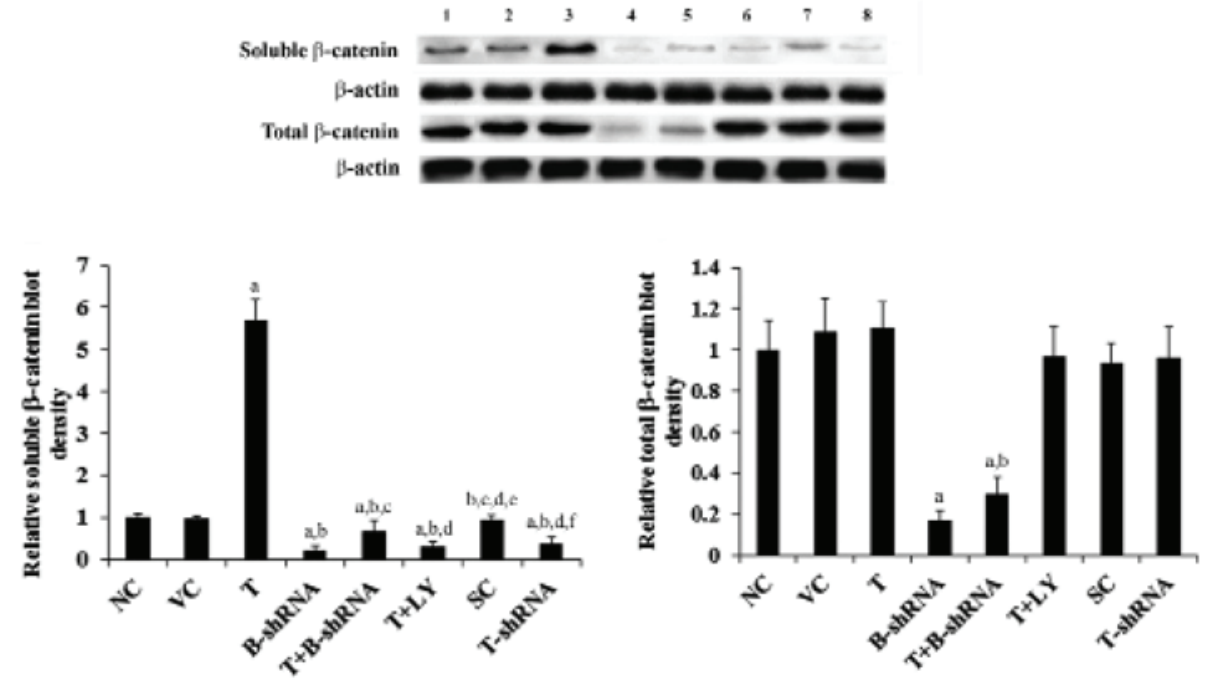

Figure 4. Effect of TWIST on levels of $\beta$-catenin protein in OTSCC cells. (A) SCC-4 and (B) TCA8113 human OTSCC cells. Lane 1, NC; lane 2, VC; lane 3 , T; lane 4, B-shRNA; lane 5, T+B-shRNA; lane 6, T+LY; lane 7, SC; lane 8, T-shRNA. The soluble and total $\beta$-catenin protein levels were analyzed with western blot analysis. $\beta$-actin was used as a loading control. The density of the $\beta$-catenin blot was normalized against that of $\beta$-actin to obtain a relative blot density, which was expressed as a fold change to the relative $\beta$-catenin blot density in the NC group (designated as 1 ). ${ }^{a} \mathrm{P}<0.05$, compared with $\mathrm{NC}$ or $\mathrm{VC}$, ${ }^{b} \mathrm{P}<0.05$, compared with $\mathrm{T},{ }^{\mathrm{C}} \mathrm{P}<0.05$, compared with $\mathrm{B}$-shRNA, ${ }^{\mathrm{d}} \mathrm{P}<0.05$, compared with $\mathrm{T}+\mathrm{B}$-shRNA, ${ }^{\mathrm{e}} \mathrm{P}<0.05$, compared with $\mathrm{T}+\mathrm{LY}$ and ${ }^{\mathrm{f}} \mathrm{P}<0.05$, compared with SC. OTSCC, oral tongue squamous cell carcinoma; NC, normal control; VC, cells transfected with empty pcDNA3 vector; T, cells transfected with pcDNA3-TWIST expression vector; T+B-shRNA, cells transfected with pcDNAs-TWIST expression vector and $\beta$-catenin short hairpin RNA; T+Ly, cells transfected with pcDNAs-WIST expression vector and treated with $50 \mu \mathrm{m} \mathrm{LY294002;} \mathrm{SC,} \mathrm{cells} \mathrm{transfected} \mathrm{with} \mathrm{scrambled} \mathrm{control} \mathrm{shRNA;} \mathrm{T-shRNA,} \mathrm{cells}$ transfected with TWIST shRNA; TWIST, twist-related protein 1. 
A
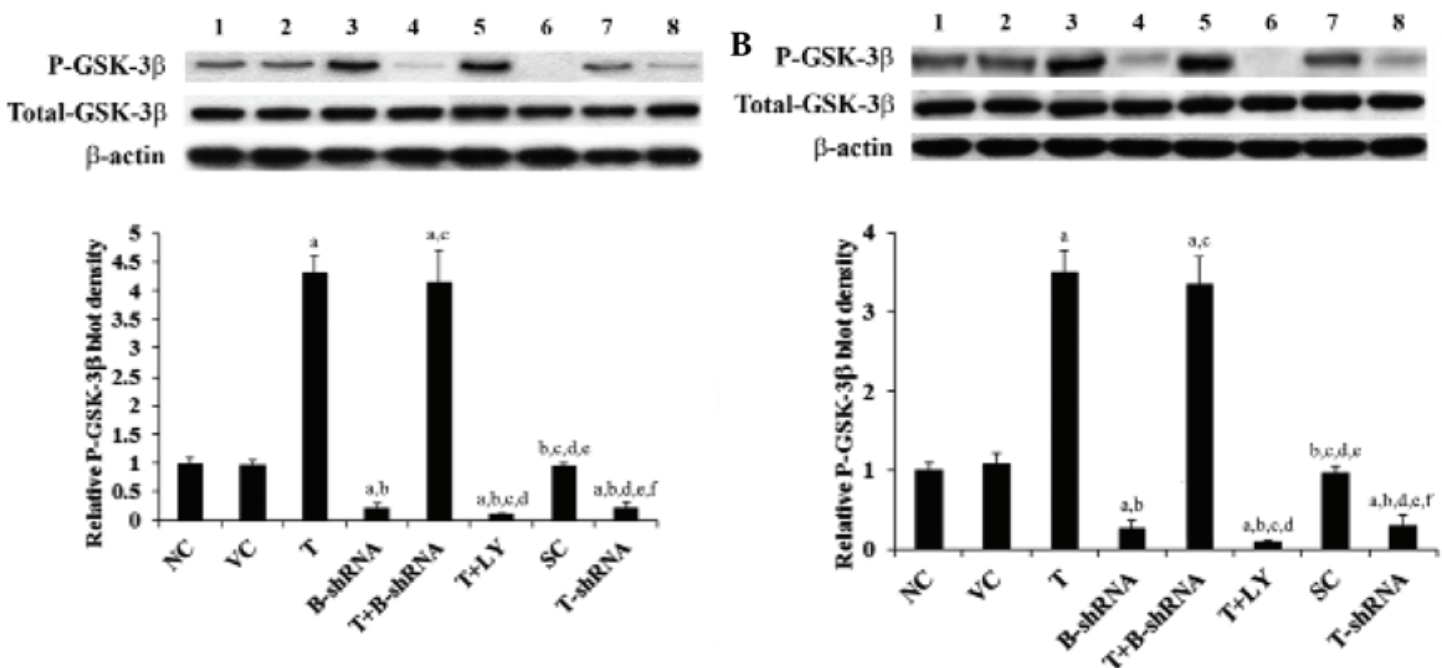

Figure 5. Effect of TWIST on phosphorylated GSK-3 $\beta$ levels in OTSCC cells. (A) SCC-4 and (B) TCA8113 human OTSCC cells. Lane 1, NC; lane 2, VC; lane 3, T; lane 4, B-shRNA; lane 5, T+B-shRNA; lane 6, T+LY; lane 7, SC; lane 8, T-shRNA. Phosphorylation of GSK-3 $\beta$ at serine 9 in each group was analyzed with western blotting. $\beta$-actin was used as a loading control. The density of the P-GSK-3 $\beta$ blot was normalized against that of total GSK-3 $\beta$ and $\beta$-actin to obtain a relative blot density, which was expressed as a fold change of the relative P-GSK-3 $\beta$ blot density in the NC group (designated as 1 ). ${ }^{\mathrm{a}} \mathrm{P}<0.05$, compared with $\mathrm{NC}$ or $\mathrm{VC},{ }^{b} \mathrm{P}<0.05$, compared with $\mathrm{T},{ }^{\mathrm{C}} \mathrm{P}<0.05$, compared with $\mathrm{B}$-shRNA, ${ }^{\mathrm{d}} \mathrm{P}<0.05$, compared with $\mathrm{T}+\mathrm{B}$-shRNA, ${ }^{\mathrm{e}} \mathrm{P}<0.05$, compared with $\mathrm{T}+\mathrm{LY}$ and ${ }^{f} \mathrm{P}<0.05$, compared with SC. P-GSK-3 $\beta$, phosphorylated glycogen synthase- $3 \beta$; OTSCC, oral tongue squamous cell carcinoma; NC, normal control; VC, cells transfected with empty pcDNA3 vector; T, cells transfected with pcDNA3-TWIST expression vector; T+B-shRNA, cells transfected with pcDNAs-TWIST expression vector and $\beta$-catenin short hairpin RNA; T+Ly, cells transfected with pcDNAs-WIST expression vector and treated with $50 \mu \mathrm{m} \mathrm{LY294002;} \mathrm{SC,} \mathrm{cells}$ transfected with scrambled control shRNA; T-shRNA, cells transfected with TWIST shRNA; TWIST, twist-related protein 1.

been stably transfected with TWIST. These results were not altered by transduction of $\beta$-catenin-shRNA (Fig. 1).

SCC-4 and TCA8113 cells overexpressing TWIST show an increase in $\beta$-catenin transcriptional activity. As shown in Fig. 2 , the transcriptional activity of $\beta$-catenin signaling in SCC-4 and TCA8113 cells was measured with TOPflash, a synthetic $\beta$-catenin/Tcf-dependent luciferase reporter (11). Compared with controls, the luciferase activity of TOPflash was increased seven-fold in SCC-4 and TCA8113 cells overexpressing TWIST. This effect was eliminated in cells stably transduced with $\beta$-catenin-shRNA. By contrast, knockdown of TWIST decreased the luciferase activity of TOPflash by $\sim 70 \%$ (Fig. 2). However, little change was observed with FOPflash, a negative control reporter with a mutation in the Tcf binding elements (11) (Fig. 2). These results suggest that TWIST may regulate $\beta$-catenin signaling in OTSCC cells.

TWIST increases $m R N A$ levels of target genes of $\beta$-catenin signaling. As shown in Fig. 3, RT-qPCR demonstrated that overexpression or knockdown of TWIST had no significant effect on $\beta$-catenin mRNA levels in SCC-4 and TCA8113 cells. However, the mRNA levels of target genes (c-Myc and c-Jun) of $\beta$-catenin signaling were increased 4.5 -fold in cells overexpressing TWIST. Again, this effect was abrogated by transduction of $\beta$-catenin-shRNA and by the phosphatidylinositol 3-kinase (PI3K) inhibitor, LY294002. Knockdown of TWIST decreased the mRNA levels of c-Myc and c-Jun by $\sim 60 \%$, compared with the control (Fig. 3).

TWIST increases levels of soluble, but not of total, $\beta$-catenin protein levels. As shown in Fig. 4 , total $\beta$-catenin protein levels in SCC-4 and TCA8113 cells were not altered by overexpression or knockdown of TWIST. By contrast, overexpression of TWIST increased the levels of soluble $\beta$-catenin by 5.5 -fold. This effect was eliminated by transduction of $\beta$-catenin-shRNA and by administration of LY294002. Knockdown of TWIST decreased the soluble $\beta$-catenin level by over $60 \%$ (Fig. 4).

TWIST increases levels of soluble $\beta$-catenin by increasing the phosphorylation of GSK-3 $\beta$. GSK-3 $\beta$ is a major downstream target of the PI3K/Akt pathway. It is inactivated by phosphorylation at serine 9 by PI3K/Akt. This results in the stabilization and accumulation of soluble $\beta$-catenin (16). As shown in Fig. 5, the total GSK-3 $\beta$ protein level in SCC-4 and TCA8113 cells was not altered by overexpression or knockdown of TWIST. Overexpression of TWIST increased phosphorylation of GSK-3 $\beta$ at serine 9 by 3.5 -fold. This effect was eradicated by administration of LY294002, although not by transduction of $\beta$-catenin-shRNA. Knockdown of TWIST decreased serine 9 phosphorylation by $\sim 70 \%$ (Fig. 5). These results indicate that TWIST is able to regulate levels of soluble $\beta$-catenin via induction of phosphorylation of GSK-3 $\beta$ by PI3K/Akt in OTSCC cells.

TWIST overexpression increases OTSCC cell invasion and the expression of MMP-2. TWIST and $\beta$-catenin are important for OTSCC cell invasion $(6,12,13)$. MMPs are also known to be involved in cancer cell invasion $(17,18)$. The effect of TWIST and $\beta$-catenin on OTSCC cell invasion and MMP expression was investigated. As shown in Fig. 6, overexpression of TWIST markedly increased SCC-4 and TCA8113 cell invasiveness. This effect was abrogated by transduction with $\beta$-catenin-shRNA and by administration of LY294002. By contrast, knockdown of TWIST markedly decreased cell 
A

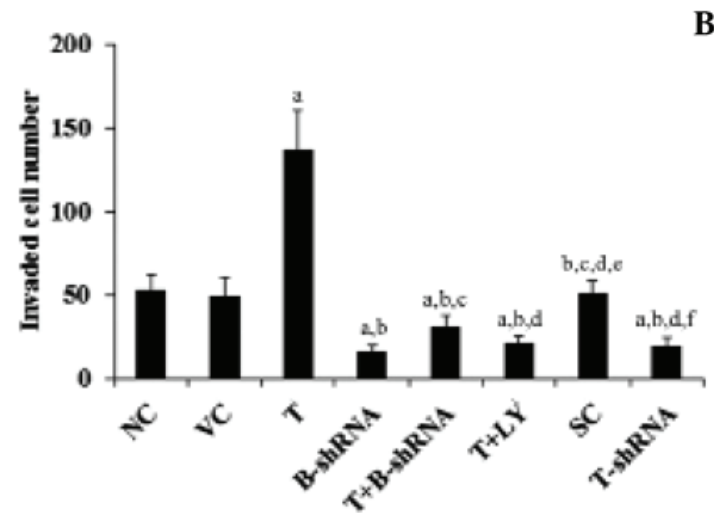

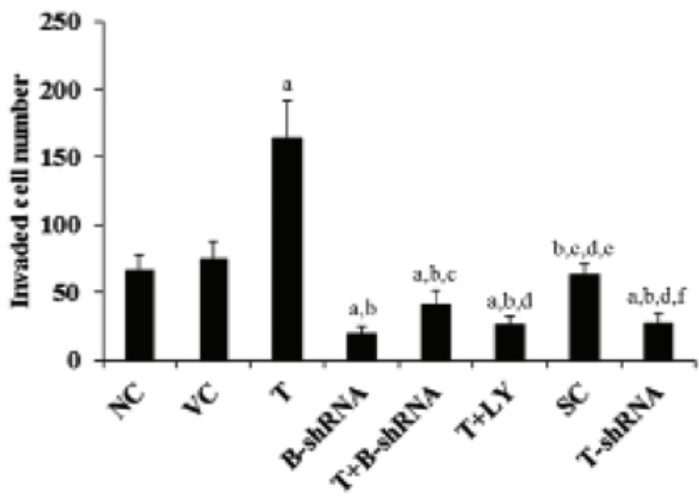

Figure 6. Effect of TWIST on OTSCC cells. (A) SCC-4 and (B) TCA8113 human OTSCC cells. Transwell invasion assays were performed in each group and the number of cells that had invaded were counted. ${ }^{\mathrm{a}} \mathrm{P}<0.05$, cpmpared with $\mathrm{NC}$ or $\mathrm{VC},{ }^{\mathrm{b}} \mathrm{P}<0.05$, compared with $\mathrm{T},{ }^{\mathrm{c}} \mathrm{P}<0.05$, compared with $\mathrm{B}$-shRNA, ${ }^{\mathrm{d}} \mathrm{P}<0.05$, compared with $\mathrm{T}+\mathrm{B}-$ shRNA, ${ }^{\mathrm{e}} \mathrm{P}<0.05$, compared with $\mathrm{T}+\mathrm{LY}$ and ${ }^{\mathrm{f}} \mathrm{P}<0.05$, compared with $\mathrm{SC}$. OTSCC, oral tongue squamous cell carcinoma; $\mathrm{NC}$, normal control; VC, cells transfected with empty pcDNA3 vector; T, cells transfected with pcDNA3-TWIST expression vector; T+B-shRNA, cells transfected with pcDNAs-TWIST expression vector and $\beta$-catenin short hairpin RNA; T+Ly, cells transfected with pcDNAs-WIST expression vector and treated with $50 \mu \mathrm{m}$ LY294002; SC, cells transfected with scrambled control shRNA; T-shRNA, cells transfected with TWIST shRNA; TWIST, twist-related protein 1.
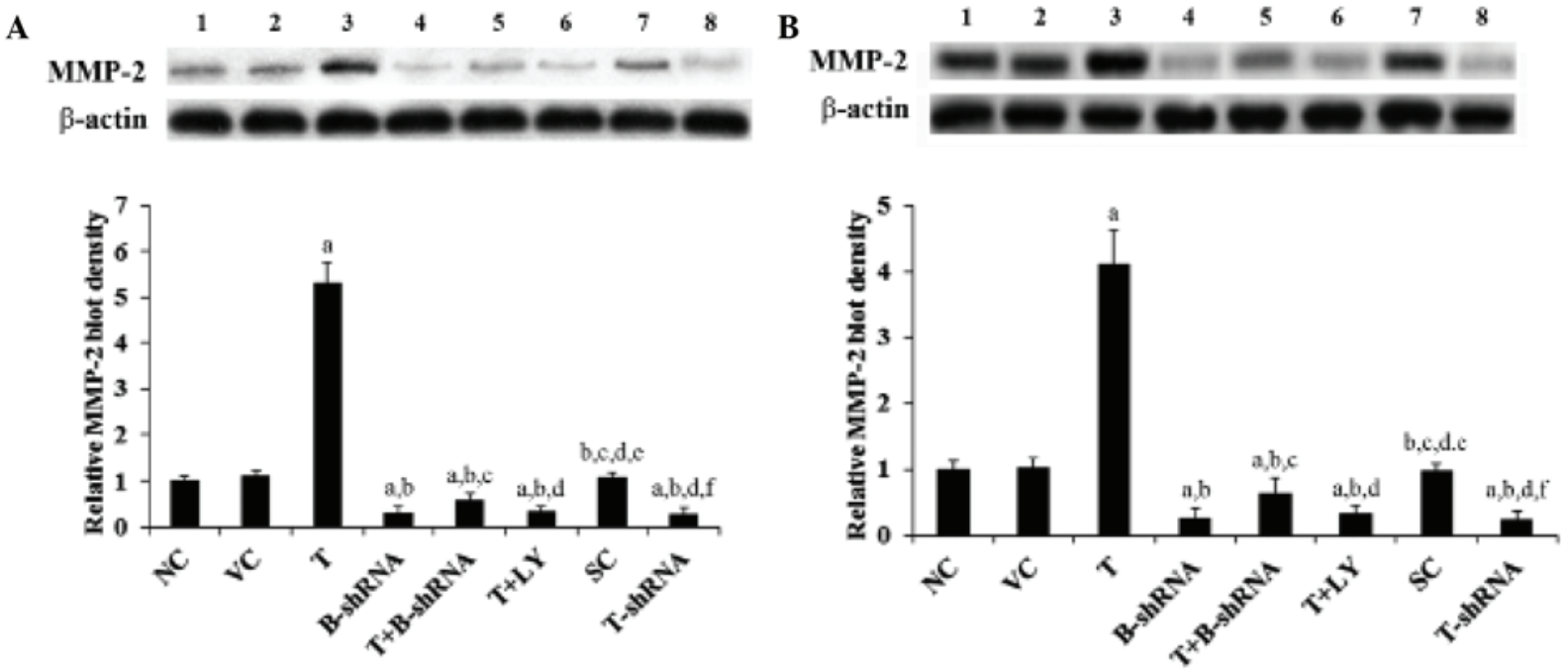

Figure 7. Effect of TWIST on expression of MMP-2 in OTSCC cells. (A) SCC-4 and (B) TCA8113 human OTSCC cells. Lane 1, NC; lane 2, VC; lane 3 , T; lane 4, B-shRNA; lane 5, T+B-shRNA; lane 6, T+LY; lane 7, SC; lane 8, T-shRNA. The expression of MMP-2 in each group was measured using western blot analysis. $\beta$-actin was used as a loading control. The density of the MMP-2 blot was normalized against that of $\beta$-actin to obtain a relative blot density, which was expressed as a fold change of the relative MMP-2 blot density in the $\mathrm{NC}$ group (designated as 1 ). ${ }^{\mathrm{a}} \mathrm{P}<0.05$, compared with $\mathrm{NC}$ or $\mathrm{VC}$, ${ }^{\mathrm{b}} \mathrm{P}<0.05$, compared with $\mathrm{T},{ }^{\mathrm{C}} \mathrm{P}<0.05$, compared with B-shRNA, ${ }^{\mathrm{d}} \mathrm{P}<0.05$, compared with $\mathrm{T}+\mathrm{B}$-shRNA, ${ }^{\mathrm{e}} \mathrm{P}<0.05$, compared with $\mathrm{T}+\mathrm{LY}$ and ${ }^{\mathrm{f}} \mathrm{P}<0.05$, compared with $\mathrm{SC}$. MMP-2, matrix metalloproteinase-2, OTSCC, oral tongue squamous cell carcinoma; NC, normal control; VC, cells transfected with empty pcDNA3 vector; T, cells transfected with pcDNA3-TWIST expression vector; T+B-shRNA, cells transfected with pcDNAs-TWIST expression vector and $\beta$-catenin short hairpin

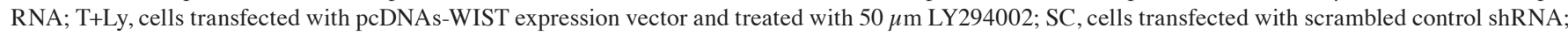
T-shRNA, cells transfected with TWIST shRNA; TWIST, twist-related protein 1.

invasion (Fig. 6). In accordance with these findings, overexpression of TWIST led to an increase in MMP-2 expression, whilst knockdown of TWIST led to a reduction in MMP-2 expression compared with controls (Fig. 7).

\section{Discussion}

Accumulating in vitro evidence suggests that $\beta$-catenin signaling is important in OTSCC cell invasion $(12,13)$. Abnormal activation of TWIST has been implicated in several types of human cancer (19). A recent study has reported that overexpression of TWIST is associated with a poor prognosis in patients with OTSCC, and may enhance OTSCC cell invasion (6). To the best of our knowledge, this study provides the first evidence that TWIST enhances OTSCC cell invasion through regulation of $\beta$-catenin signaling. The data were highly consistent in the two OTSCC cell lines.

In this study, overexpression and knockdown of TWIST in OTSCC cells increased and decreased the levels of soluble $\beta$-catenin, respectively. $\beta$-catenin interacts with Tcf transcription factors to activate a number of downstream target genes, including c-Myc and c-Jun $(8,10,11)$. Overexpression and knockdown of TWIST increased and decreased, respectively, the TOPflash $\beta$-catenin signaling reporter activity, as well as 
the mRNA levels of c-Myc and c-Jun. Notably, TWIST did not alter the total levels of the $\beta$-catenin protein, indicating that TWIST may regulate the level of soluble $\beta$-catenin via a post-transcriptional mechanism. This is in accordance with the observed increase in serine- 9 phosphorylation of GSK-3 $\beta$, which ultimately results in stabilization and accumulation of soluble $\beta$-catenin (16). GSK-3 $\beta$ is a major downstream target of the PI3K/Akt pathway (16). Since the PI3K inhibitor, LY294002, eliminated the increase in serine 9 phosphorylation of GSK-3 $\beta$ and the increase in soluble $\beta$-catenin induced by overexpression of TWIST, it is likely that TWIST regulates the soluble $\beta$-catenin level in OTSCC cells through the PI3K/Akt/GSK-3 $\beta$ pathway.

Overexpression of TWIST markedly enhanced cell invasion and MMP-2 expression in OTSCC cells. This result was corroborated by a significant reduction in cell invasion and MMP-2 expression in OTSCC cells with the knockdown of TWIST. The enhancing effect of TWIST on OTSCC cell invasion and MMP-2 expression was almost completely eradicated by knocking down $\beta$-catenin with shRNA, suggesting that $\beta$-catenin signaling is an essential mediator of the effect of TWIST on OSTCC cell invasion.

Abnormal activation of $\beta$-catenin signaling is critical in the progression of a variety of cancers, including OTSCC (11-13). Yin et al (15) showed that TWIST negatively regulated $\beta$-catenin signaling via a PI3K-dependent mechanism in osteosarcoma cells. Their findings are in accordance with the fact that in a homogeneous cohort of osteosarcoma patients, the TWIST gene is frequently found to be deleted in the tumors at diagnosis, and haploinsufficiency of this gene is significantly correlated with a poorer patient outcome (15). The present study, however, found that TWIST was a positive regulator of $\beta$-catenin signaling by a PI3K-dependent mechanism. This finding is in agreement with a recent study demonstrating that overexpression of TWIST is associated with a poor prognosis in patients with OTSCC (6). This discrepancy suggests that the regulatory effect of TWIST on $\beta$-catenin signaling may be dependent on the type of tissue or cancer involved.

MMPs are critical for cancer cell invasion $(17,18)$. Recent studies have suggested that MMP-2 is important for OTSCC lymph node metastasis in vivo and OTSCC cell invasion in vitro $(6,20)$. This study found that TWIST markedly increased MMP-2 expression through $\beta$-catenin signaling, suggesting that the TWIST/ $\beta$-catenin signaling axis is important for OTSCC progression. In addition, as TWIST and $\beta$-catenin signaling are abnormally activated in a variety of cancers, the TWIST/ $\beta$-catenin signaling axis may be important in cancers other than OTSCC. This hypothesis requires further investigation in future studies.

In conclusion, the current study demonstrated that TWIST enhances cell invasion and MMP-2 expression in OTSCC cells through its effects on $\beta$-catenin signaling, which are likely to be mediated via a PI3K-dependent mechanism. This study provides novel insights into the molecular mechanisms underlying OTSCC progression.

\section{References}

1. Choi KK, Kim MJ, Yun PY, Lee JH, Moon HS, Lee TR and Myoung H: Independent prognostic factors of 861 cases of oral squamous cell carcinoma in Korean adults. Oral Oncol 42: 208-217, 2006.

2. Xing Y, Qi J, Deng S, Wang C, Zhang L and Chen J: Small interfering RNA targeting ILK inhibits metastasis in human tongue cancer cells through repression of epithelial-to-mesenchymal transition. Exp Cell Res 319: 2058-2072, 2013.

3. Su HH, Chu ST, Hou YY, Chang KP and Chen CJ: Spindle cell carcinoma of the oral cavity and oropharynx: factors affecting outcome. J Chin Med Assoc 69: 478-483, 2006.

4. Entz-Werlé N, Lavaux T, Metzger N, et al: Involvement of MET/TWIST/APC combination or the potential role of ossification factors in pediatric high-grade osteosarcoma oncogenesis. Neoplasia 9: 678-688, 2007.

5. Entz-Werlé N, Choquet P, Neuville A, et al: Targeted apc;twist double-mutant mice: a new model of spontaneous osteosarcoma that mimics the human disease. Transl Oncol 3: 344-353, 2010.

6. da Silva SD, Alaoui-Jamali MA, Soares FA, et al: TWIST1 is a molecular marker for a poor prognosis in oral cancer and represents a potential therapeutic target. Cancer 120: 352-362, 2014.

7. Chesire DR and Isaacs WB: Beta-catenin signaling in prostate cancer: an early perspective. Endocr Relat Cancer 10: 537-560, 2003.

8. Cawthorn WP, Heyd F, Hegyi K and Sethi JK: Tumour necrosis factor-alpha inhibits adipogenesis via a beta-catenin/TCF4(TCF7L2)-dependent pathway. Cell Death Differ 14: 1361-1373, 2007.

9. Nusse R: WNT targets. Repression and activation. Trends Genet 15: 1-3, 1999.

10. Gan XQ, Wang JY, Xi Y, Wu ZL, Li YP and Li L: Nuclear Dvl, c-Jun, beta-catenin, and TCF form a complex leading to stabilization of beta-catenin-TCF interaction. J Cell Biol 180: 1087-1100, 2008

11. Sun P, Xiong H, Kim TH, Ren B and Zhang Z: Positive inter-regulation between beta-catenin/T cell factor- 4 signaling and endothelin- 1 signaling potentiates proliferation and survival of prostate cancer cells. Mol Pharmacol 69: 520-531, 2006.

12. Wang LP, Chen SW, Zhuang SM, Li H and Song M: Galectin-3 accelerates the progression of oral tongue squamous cell carcinoma via a Wnt $/ \beta$-catenin-dependent pathway. Pathol Oncol Res 19: 461-474, 2013.

13. Kawakita A, Yanamoto S, Yamada SI, Naruse T, Takahashi H, Kawasaki G and Umeda M: MicroRNA-21 promotes oral cancer invasion via the $\mathrm{Wnt} / \beta$-catenin pathway by targeting DKK2. Pathol Oncol Res, Sep 3, 2013 (Epub ahead of print).

14. Matsuo N, Shiraha H, Fujikawa T, et al: Twist expression promotes migration and invasion in hepatocellular carcinoma. BMC Cancer 9: 240, 2009.

15. Wu J, Liao Q, He H, Zhong D and Yin K: TWIST interacts with $\beta$-catenin signaling on osteosarcoma cell survival against cisplatin. Mol Carcinog 53: 440-446, 2014.

16. Sharma M, Chuang WW and Sun Z: Phosphatidylinositol 3-kinase/Akt stimulates androgen pathway through GSK3beta inhibition and nuclear beta-catenin accumulation. J Biol Chem 277: 30935-30941, 2002.

17. Fan HX, Li HX, Chen D, Gao ZX and Zheng JH: Changes in the expression of MMP2, MMP9, and ColIV in stromal cells in oral squamous tongue cell carcinoma: relationships and prognostic implications. J Exp Clin Cancer Res 31: 90, 2012.

18. Li H, Song H, Luo J, Liang J, Zhao S and Su R: Knockdown of glucose-regulated protein 78 decreases the invasion, metalloproteinase expression and ECM degradation in hepatocellular carcinoma cells. J Exp Clin Cancer Res 31: 39, 2012.

19. Entz-Werlé N, Stoetzel C, Berard-Marec P, et al: Frequent genomic abnormalities at TWIST in human pediatric osteosarcomas. Int J Cancer 117: 349-355, 2005.

20. Xiao W, Jiang M, Li H, Li C, Su R and Huang K: Knockdown of FAK inhibits the invasion and metastasis of Tca-8113 cells in vitro. Mol Med Rep 8: 703-707, 2013. 